\title{
Endobronchial argon plasma coagulation for the management of postintubation tracheal stenosis
}

by

Masanori Yasuo*, Tsuyoshi Tanabe*, Kenji Tsushima*, Masaru Nakamura*, Shintaro Kanda*, Seiichi Yamazaki*, Michiko Ito*, Shino Furuya*, Sumiko Yoshikawa*, Keishi Kubo*, Satoshi Kawakami**, and Yoshitaka Yamazaki***

* First Department of Internal Medicine, Shinshu University School of Medicine,

** Department of Radiology, Shinshu University School of Medicine,

***Department of Endoscopy, Shinshu University School of Medicine, Matsumoto, JAPAN

Short title: Endobronchial argon plasma coagulation

Correspondence: Masanori Yasuo

$1^{\text {st }}$ Department of Internal Medicine

Shinshu University School of Medicine

3-1-1 Asahi, Matsumoto, Japan

Telephone: +8126337 2631, Fax: +81263363722

E-mail address: yasumasa@hsp.md.shinshu-u.ac.jp

Words: 1684 words 


\begin{abstract}
Postintubation tracheal stenosis is usually caused by pressure necrosis at the cuff. Despite the fact that this phenomenon is well known and both large volume and low pressure cuffs have been developed, this lesion nevertheless continues to occur. Although the best results are obtained at a tracheal reconstruction by an experienced surgeon, not all patients are able to undergo this operation for either medical or personal reasons. Argon plasma coagulation (APC) using flexible bronchoscopy has been successfully employed in the treatment of postintubation tracheal stenosis in two of the surgery-refused and inoperable patients. The patients immediately experienced a relief of symptoms after APC. APC was thus performed 3 to 4 times every one to two weeks for each patient. In addition, there were no complications related to this procedure. The number of published clinical reports describing APC in benign airway stenosis are increasing. APC has also been reported to have several advantages over other interventional endobronchial techniques in the management of tracheo-bronchial stenosis. To our knowledge, this is the first description of APC being used in the treatment of endobronchial dilatation against postintubation tracheal stenosis.
\end{abstract}

Key words: airway obstruction, flexible bronchoscopy, argon plasma coagulation, endobronchial therapy, electrocoagulation 


\section{Introduction}

Postintubation tracheal stenosis is still a serious problem because it can directly result in death. The etiology of this disease has gradually becomes clear and most such injuries are considered to be caused by pressure necrosis [1] Despite the fact that numerous improvements have been made in both the endotracheal tubes and cuff management related with ventilator therapy, these lesions still continue to occur. A resection and reconstruction seems to be the best choice for the management of postintubation tracheal stenosis. Grillo et al. has reported quite good results (93.7\% successful) in a total of 471 patients of tracheal resection and reconstruction [2]. However, not all patients could undergo surgery due to medical and/or personal reasons, etc.

Argon plasma coagulation (APC) is a form of noncontact electrocoagulation which has been used for open surgery for more than 15 years [3], as well as for both laparoscopy [4] and gastro-intestinal endoscopy [5]. It has recently been widely used in tracheobronchial disease $[6,7]$ because it allows a rapid coagulation with minimal manipulation and mechanical trauma to the target tissue [7]. Although APC has been proven to be effective for the treatment of endoluminal hemoptysis [7], benign and malignant airway narrowing [7] and post-tracheal anastomosis [8], the use of this treatment modality for postintubation tracheal stenosis has not yet been reported. We herein discuss that the successful use of APC in the management of postintubation tracheal stenosis.

\section{Case Report}

Case 1

A 74-year-old woman was admitted to our hospital for tracheoplasty. She had previously undergone bilateral total knee arthroplasty to treat gonarthrosis at another hospital. Two hours after arthroplasty, she suffered a pulmonary embolism and had thus been treated with mechanical ventilation for 15 days. Eight weeks later, severe concentric cicatrical tracheal stenosis developed. Because surgery was refused and because the three-dimensional CT (Fig 1A) revealed a relatively short length of the lesion, we decided to dilate the tracheal stenosis with APC using a flexible bronchoscope. Bronchoscopy revealed a pinhole stenosis of the trachea 3 $\mathrm{cm}$ below the vocal cord (Fig 1B). We performed several short bursts of APC at the sites of stenosis, and the devitalized tissue was then mechanically removed with grasping forceps. Her respiratory distress and oxygen saturation both immediately subsided after the first dilation (Fig 1C). The APC procedure was performed 3 
times to devitalize the granulomatous tissue. After the third APC treatment (2 months after the first APC treatment), the stenotic site was finally dilated to $7 \mathrm{~mm}$ in diameter and she has not experienced either re-stenosis and respiratory distress up until now (5 months after the first APC treatment, Fig 1D). Because the original diameter at the context of her trachea was $10 \mathrm{~mm}$ from CT, the degree of the dilatation is thus considered to be a permissible size.

\section{Case 2}

A 72-year-old woman had been ventilated via an endotracheal tube 7 days for severe respiratory failure secondary to pneumonia. She had a chronic respiratory failure due to sequelae of tuberculosis. Her endotracheal tube was changed to a tracheal cannula from a tracheal stoma and then it was to a changed silicone formed tracheal retainer (Tracheal Opening Retainer ${ }^{\circledR}$, KOKEN, Tokyo, Japan) at 3 weeks after mechanical ventilation from tracheal stoma. Two weeks after mechanical ventilation (i.e. at 6 weeks after endotracheal intubation), she had difficulty in coughing up phlegm. Bronchoscopy revealed a pinhole stenosis of the trachea at $2.8 \mathrm{~cm}$ from the upper carina. Surgery seemed to be difficult because of a low respiratory function and severe pulmonary adhesion. The woman came to our hospital to undergo endobronchial dilatation. The dilatation by APC and the mechanical removal of the devitalized tissue with grasping forceps was successfully performed and her symptoms were thus relieved. The above combined dilatation was performed once and then APC treatment was carried out another three times. As a result, a permissible dilatation was therefore achieved (two months after the first APC treatment, Fig 1E). Computed tomography revealed the site of stenosis to be dilated to a diameter of $8.3 \mathrm{~mm}$. Because the original diameter at the context of her trachea was $11 \mathrm{~mm}$ from CT (Fig 1F), the degree of the dilatation is thus considered to be a permissible size. No re-stenosis was observed in the subsequent follow-up bronchoscopy.

\section{Methods}

Endobronchial APC procedure was performed with a flexible bronchoscope. Local anesthesia and conscious sedation with IM pethidine hydrochloride and atropine was administered before the procedure. Topical anesthesia was achieved by endobronchial instillation of $2 \%$ lidocaine. The pulse oximetry saturation, resting pulse and electrocardiograms were monitored during bronchoscopy. The equipment for APC consists of the APC probe, an argon-gas source, and a 
high-frequency surgical unit (APC 300, ERBOTOM ICC; Erbe Elektromedizin; Tuebingen, Germany). To deliver the gas, we used a flexible-monopolar Teflon tube with a $1.5-\mathrm{mm}$ diameter, $150-\mathrm{cm}$ length; this was put into the working channel of the flexible bronchoscope. Energy at $30 \mathrm{~W}$ and $\operatorname{argon}$ flow at $0.5 \mathrm{~L} / \mathrm{min}$ were then applied to the Teflon tube. The stenotic lesion was endoscopically visualized and then coagulated from the site of the stenosis. The time it took to perform each coagulation was about 1 to 2 seconds, and coagulation was repeatedly performed at the stenotic site. When such coagulation was done several times at the stenotic site, then the site gradually became dilated. Thereafter, the devitalized tissue was mechanically removed with grasping forceps. We did not use any dilation device such as the neodymium-yttrium aluminium garnet (Nd-YAG) laser or balloon dilation. A series of repetitive coagulations and removal of devitalized tissue was done repeatedly until satisfactory dilatation (about $5 \mathrm{~mm}$ diameter) was achieved. After about 48 hours, the devitalized tissue was spontaneously detached that accompanied tissue necrosis, and then the tracheal lumen was satisfactorily dilated (about 8 to $10 \mathrm{~mm}$ diameter). Thereafter surveillance bronchoscopy was performed from 14 to 28 days after therapy and as clinically indicated. 


\section{Discussion}

In this study, our experience in performing APC of postintubation tracheal stenosis is presented. The APC enabled us to safely create tracheal dilatation in two patients with severe postintubation tracheal stenosis in whom reconstructive surgery was refused or the surgery was considered too dangerous because of a poor pulmonary function and severe pulmonary adhesion.

The use of high volume, low pressure cuffs is now standard practice at intensive care units. However, postintubation tracheal stenosis may still occur. Studies into stenosis at the cuff site have shown the main cause to be the pressure exerted on the tracheal mucosa by the cuff [9].

As Simpson et al. mentioned [10] from 60 cases experience in the endoscopic management of laryngo-tracheal stenosis, purely endoscopic techniques are unsuccessful in most cases of severe tracheal stenosis especially if the stenotic areas are wide, circumferential, or if they are accompanied by a significant loss of cartilage in the trachea. Therefore, a resection and reconstruction is the mainstay of treatment for the management of postintubation tracheal stenosis, especially in cases of extensive tracheal stenosis [10]. However there are some patients in whom a surgical resection and end-to-end anastomosis are contraindicated, not feasible, or refused. Because the progression of endobronchial therapy is evident $[5-8,10-12]$, endobronchial management thus seems to be a satisfactory treatment for the management of postintubation tracheal stenosis. In 1999, Brichet et al [13] advocated that the optimal management of postintubation tracheal stenosis should be endobronchial dilatation by Nd-YAG laser or stent implantation for the first therapeutic choice. Furthermore, balloon dilatation, stent insertion, laser incision and several these combinations have been reported as a treatment solution for inoperable cases $[11,12]$, however, several complications have also been reported [7, 11, 14]. Especially endobronchial electrosurgery with Nd-YAG laser is considered to be an effective procedure for treating such airway-obstructive lesions [12], however, surgeons must be extremely careful to avoid extensive infiltration of the bronchial wall, otherwise Nd-YAG laser for treatment endobronchial dilatation can lead to cartilaginous damage, fibrosis, and subsequent stenosis $[12,15]$.

Recently, APC has been introduced into the field of flexible bronchoscopy, and it has been used in the treatment of benign or malignant lesions in the airway [5-8, 11]. It has an excellent safety profile, is convenient and simple to use, and is relative low cost, make it most suitable for therapeutic applications through the flexible bronchoscope [7]. Morice et al. [7] mentioned that APC devitalizes tissue 
gradually by producing temperatures that coagulate and desiccate tissue. As the target surface becomes less electrically conductive, APC automatically seeks adjacent tissue with less electrical resistance. This can thus result in a homogenous but limited depth of penetration (approximately $3 \mathrm{~mm}$ ). As a result, APC offers uniform coagulation and good protection against airway perforation [7]. As mentioned above, APC cannot dilate the tissue so deeply, therefore a relatively short length of stenosis can be adapted [7]. Both of the lesions in our cases were in short length with pinhole stenosis. The site of stenotic lesions is safely devitalized and removed through sequential APC and grasping forceps. We did not experience any crucial trouble during the seven treatment courses in our two cases. There are some points of notice regarding the APC procedure; namely, the APC probe should be extended beyond the tip of the bronchoscope to avoid thermal damage caused by the bronchoscope, and maintain the inspired oxygen concentration at $\leq 40 \%$ [7]. If we can adhere to these points, then safe and effective endobronchial dilatation can be assured.

Since we only performed APC dilatation against postintubation tracheal stenosis, we did not have any data about the risk of restenosis. On the other hand, because Brichet [13] reported that 3 out of 15 patients with pinhole stenosis had to later receive other therapies (i.e. surgical treatment and Dumon stent implantation) after performing Nd-YAG laser dilatation three times [13], we thus expected that a similar frequency of restenosis might occur in the areas undergoing APC dilatation.

In summary, we successfully performed endobronchial dilatation using APC in two cases of postintubation tracheal stenosis. Although tracheal reconstruction still remains the best treatment for this disease, APC is considered to be an effective alternative procedure for endobronchial dilatation in selected patients who are inoperable due to either medical or personal reasons. 


\section{References}

1. Grillo HC, Donahue DM. Post intubation tracheal stenosis. Semin Thorac Cardiovasc Surg. 1996; 8: 370-80.

2. Grillo HC, Donahue DM, Mathisen DJ, Wain JC, Wright CD. Postintubation tracheal stenosis. Treatment and results. J Thorac Cardiovasc Surg. 1995; 109: 486-93.

3. Quinlan DM, Naslund MJ, Brendler CB. Application of argon beam coagulation in urological surgery. J Urol. 1992; 147: 410-2.

4. Gale P, Adeyemi B, Ferrer K, Ong A, Brill AI, Scoccia B. Histologic characteristics of laparoscopic argon beam coagulation. J Am Assoc Gynecol Laparosc. 1998; 5: 19-22.

5. Grund KE, Storek D, Farin G. Endoscopic argon plasma coagulation (APC) first clinical experiences in flexible endoscopy. Endosc Surg Allied Technol. 1994; 2 : 42-6.

6. Tremblay A, Marquette $\mathrm{CH}$. Endobronchial electrocautery and argon plasma coagulation: a practical approach. Can Respir J. 2004; 11: 305-10.

7. Morice RC, Ece T, Ece F, Keus L. Endobronchial argon plasma coagulation for treatment of hemoptysis and neoplastic airway obstruction. Chest. 2001; 119: 781-7.

8. Sato M, Terada Y, Nakagawa T, Li M, Wada H. Successful use of argon plasma coagulation and tranilast to treat granulation tissue obstructing the airway after tracheal anastomosis. Chest. 2000; 118: 1829-31.

9. Cooper JD, Grillo HC. Experimental production and prevention of injury due to cuffed tracheal tubes. Surg Gynecol Obstet. 1969; 129: 1235-41.

10. Simpson GT, Strong MS, Healy GB, Shapshay SM, Vaughan CW. Predictive factors of success or failure in the endoscopic management of laryngeal and tracheal stenosis. Ann Otol Rhinol Laryngol. 1982; 91: 384-8.

11. Noppen M, Schlesser M, Meysman M, D'Haese J, Peche R, Vincken W. Bronchoscopic balloon dilatation in the combined management of postintubation stenosis of the trachea in adults. Chest. 1997; 112:1136-40.

12. Coulter TD, Mehta AC. The heat is on: impact of endobronchial electrosurgery on the need for Nd-YAG laser photoresection. Chest. 2000; 118: 516-21.

13. Brichet A, Verkindre C, Dupont J, Carlier ML, Darras J, Wurtz A, Ramon P, Marquette $\mathrm{CH}$. Multidisciplinary approach to management of postintubation tracheal stenoses. Eur Respir J. 1999; 14:796-9.

14. Sonett JR, Keenan RJ, Ferson PF, Griffith BP, Landreneau RJ. Endobronchial 
management of benign, malignant, and lung transplantation airway stenoses. Ann Thorac Surg. 1995; 59: 1417-22.

15. Verkindre C, Brichet A, Maurage CA, Ramon P, Homasson JP, Marquette CH. Morphological changes induced by extensive endobronchial electrocautery. Eur Respir J. 1999; 14: 796-9. 


\section{Figure Legends}

Figure 1 A; Three-dimensional CT revealed a relatively short length stenosis (arrowhead). B; Bronchoscopy revealed a pinhole stenosis of the trachea. $\mathrm{C}$; Forty-eight hours after the first APC treatment, the site of postintubation tracheal stenosis was fairly dilated. D; After the third APC treatment (5 months after the first APC treatment), the site of stenosis was still stably dilated. E; After the fourth APC treatment (2 months after the first APC treatment), the site of stenosis was tolerably dilated. F; A sagittal-image of the CT was performed at the same time as broncoscopy (Figure 1E). The site of postintubation tracheal stenosis $(\dagger)$ was fairly dilated $(8.3 \mathrm{~mm})$. An asterisk $(*)$ shows the site of tracheal stoma. 